\title{
Population Shape Regression From Random Design Data
}

\author{
B. C. Davis - P. T. Fletcher • E. Bullitt • S. \\ Joshi
}

Received: date / Accepted: date

\begin{abstract}
Regression analysis is a powerful tool for the study of changes in a dependent variable as a function of an independent regressor variable, and in particular it is applicable to the study of anatomical growth and shape change. When the underlying process can be modeled by parameters in a Euclidean space, classical regression techniques $[15,38]$ are applicable and have been studied extensively. However, recent work suggests that attempts to describe anatomical shapes using flat Euclidean spaces undermines our ability to represent natural biological variability [10,12].

In this paper we develop a method for regression analysis of general, manifoldvalued data. Specifically, we extend Nadaraya-Watson kernel regression by recasting the regression problem in terms of Fréchet expectation. Although this method is quite general, our driving problem is the study anatomical shape change as a function of age from random design image data.

We demonstrate our method by analyzing shape change in the brain from a random design dataset of MR images of 97 healthy adults ranging in age from 20 to 79 years. To study the small scale changes in anatomy, we use the infinite dimensional manifold of diffeomorphic transformations, with an associated metric. We regress a representative anatomical shape, as a function of age, from this population.
\end{abstract}

Keywords spatio-temporal shape analysis $\cdot$ kernel regression $\cdot$ deformable atlas building

\author{
B. C. Davis \\ University of North Carolina at Chapel Hill \\ Chapel Hill, NC, USA \\ E-mail: brad.davis@unc.edu \\ P. T. Fletcher \\ University of Utah \\ Salt Lake City, Utah, USA \\ E. Bullitt \\ University of North Carolina at Chapel Hill \\ Chapel Hill, NC, USA
}

S. Joshi

University of Utah

Salt Lake City, Utah, USA 


\section{Introduction}

An important area of medical image analysis is the development of methods for automated and computer-assisted assessment of anatomical change over time. For example, the analysis of structural brain change over time is important for understanding healthy aging. These methods also provide markers for understanding disease progression.

A number of longitudinal growth models have been developed to provide this type of analysis to time-series imagery of a single subject (e.g., $[2,8,28,36]$ ). While these methods provide important results, their use is limited by their reliance on longitudinal data, which can be impractical to obtain for many medical studies. Also, while these methods allow for the study of an individual's anatomy over time, they do not apply when the average growth for a population is of interest.

Random design data sets, which contain anatomical data from many different individuals, provide a rich environment for addressing these problems. However, in order to detect time-related trends in such data, two distinct aspects of anatomical variation must be separated: individual variation and time effect. For measurements that naturally form Euclidean vector spaces, this separation can be achieved by regressing a representative value over time from the data.

For example, in Figure 1 we apply kernel regression to measurements reported in a study by Mortamet et al. [32] on the effect of aging on gray matter and ventricle volume in the brain. The regression curves demonstrate the average volume, as a function of patient age, of these structures. These trends - on average there is a loss of gray matter and expansion of the ventricles - have been widely reported in the medical literature on aging $[13,27,32]$. While volume-based regression analysis is important, it does not provide any information about the detailed shape changes that occur in the brain, on average, as a function of age. This has motivated us to study regression of shapes.

Recent work has suggested that representing the geometry of shapes in flat Euclidean vector spaces limits our ability to represent natural variability in populations $[10,12,28]$. For example, Figure 2 demonstrates the amazing non-linear variability in brain shape among a population of healthy adults. The analysis of transformation groups that describe shape change are essential to understanding this shape variability. These groups vary in dimensionality from simple rigid rotations to the infinitedimensional group of diffeomorphisms [30]. These groups are not generally vector spaces and are instead naturally represented as manifolds.

A number of authors have contributed to the field of statistical analysis on manifolds (see Pennec [34] for a more detailed history). Early work on manifold statistics includes directional statistics $[6,19]$ and statistics of point set shape spaces $[22,23]$. The large sample properties of sample means on manifolds are developed in [4,5]. Jupp and Kent [20] describe a method of regression of spherical data that 'unwraps' the data onto a tangent plane, where standard curve fitting methods can be applied. In $[10,17,34]$, statistical concepts such as averaging and principal components analysis were extended to manifolds representing anatomical shape variability. Many of the ideas are based on the method of averaging in metric spaces proposed by Fréchet [11].

In this paper we use the notion of Fréchet expectation to generalize regression to manifold-valued data. We use this method to study spatio-temporal anatomical shape change in a random design database consisting of three-dimensional MR images of healthy adults. Our method generalizes Nadaraya-Watson kernel regression in order to compute representative images of this population over time. To determine the shape 


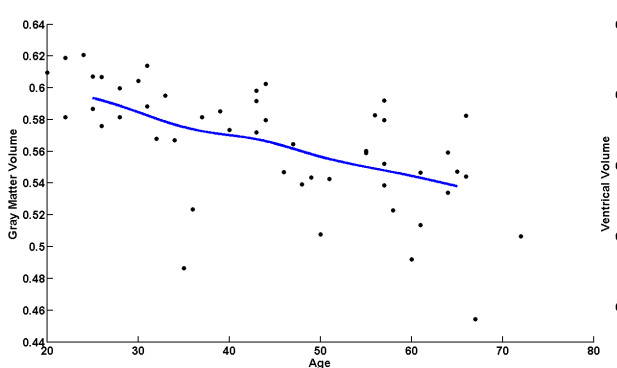

(a)

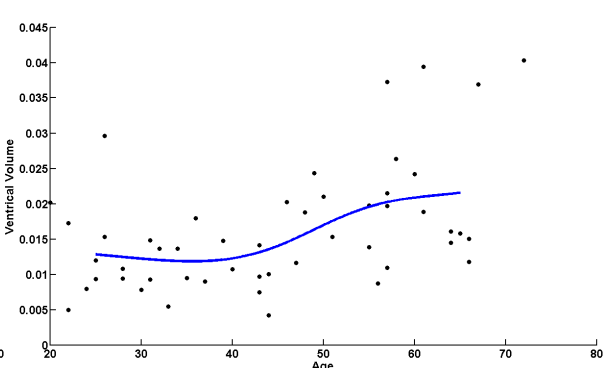

(b)

Fig. 1 Illustration of univariate kernel regression: the effect of aging on gray matter (a) and ventricle volume (b) in the brain. Circles represent volume measurements relative to total brain volume. Kernel regression is used to estimate the relationship between patient age and structure volume (filled lines).

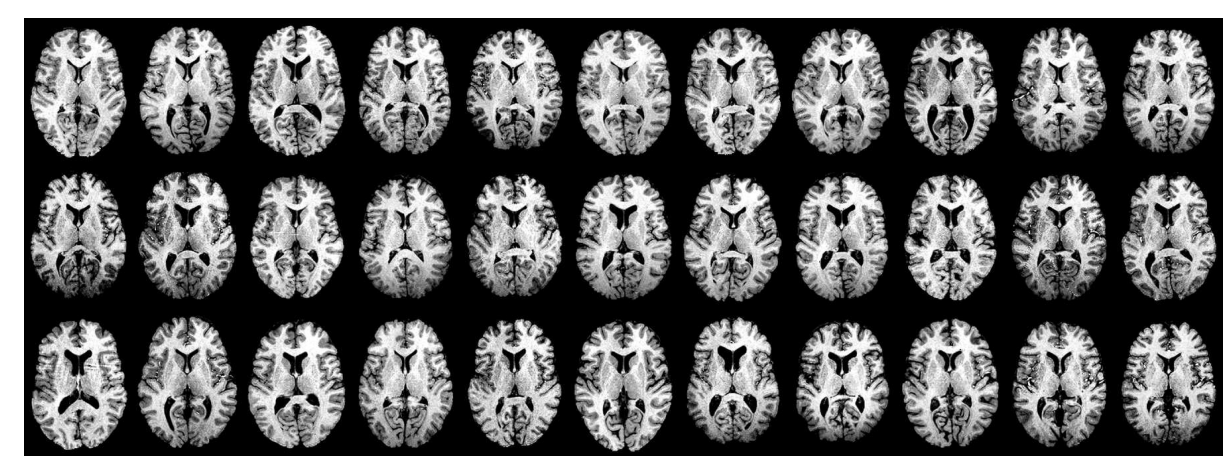

Fig. 2 Brain image database. To demonstrate the extent of natural brain shape variability within a population of healthy subjects, a mid-axial slice is presented for a sample of images used in this study. The images are arranged in order of increasing patient age from 30 (top left) to 60 (bottom right). Because of the complexity of the shapes and the high level of natural shape variability, it is extremely difficult to visually discern any patterns within these data.

change in the population over time, we apply a diffeomorphic growth model [28] to this time-indexed population representative image.

\section{Methods}

2.1 Review of univariate kernel regression

Univariate kernel regression $[15,38]$ is a non-parametric method used to estimate the relationship, on average, between an independent random variable $T$ and a dependent random variable $Y$. The estimation is based on a set of observations $\left\{t_{i}, y_{i}\right\}_{i=1}^{N}$ drawn from the joint distribution of $T$ and $Y$. This relationship between $T$ and $Y$ can be modeled as $y_{i}=m\left(t_{i}\right)+\epsilon_{i}$, where $\epsilon_{i}$ describes the random error of the model for the $i$ th observation and $m$ is the unknown function that is to be estimated. 
In this setting, $m(t)$ is defined by the conditional expectation

$$
m(t) \equiv E(Y \mid T=t)=\int y \frac{f(t, y)}{f_{T}(t)} d y
$$

where $f_{T}(t)$ is the marginal density of $T$ and $f(t, y)$ is the joint density function of $T$ and $Y$. For random design data, both $f(t, y)$ and $f_{T}(t)$ are unknown and so $m$ has no closed-form solution. A number of estimators for $m$ have been proposed in the kernel regression literature.

One such estimator - the Nadaraya-Watson kernel regression estimator [33,39] can be derived from (1) by replacing the unknown densities with their kernel density estimates

$$
\hat{f}_{T}^{h}(t) \equiv \frac{1}{N} \sum_{i=1}^{N} K_{h}\left(t-t_{i}\right) \quad \text { and } \quad \hat{f}^{h, g}(t, y) \equiv \frac{1}{N} \sum_{i=1}^{N} K_{h}\left(t-t_{i}\right) K_{g}\left(y-y_{i}\right) .
$$

In these equations, $K$ is a function that satisfies $\int_{\mathbb{R}} K(t) d t=1 . K_{h}(t) \equiv \frac{1}{h} K\left(\frac{t}{h}\right)$ and $K_{g}(t) \equiv \frac{1}{g} K\left(\frac{t}{g}\right)$ are kernel functions with bandwidths $h$ and $g$ respectively.

Plugging these density estimates into equation 1 gives

$$
\hat{m}_{h, g}(t)=\int y \frac{\frac{1}{N} \sum_{i=1}^{N} K_{h}\left(t-t_{i}\right) K_{g}\left(y-y_{i}\right)}{\frac{1}{N} \sum_{i=1}^{N} K_{h}\left(t-t_{i}\right)} d y .
$$

Finally, assuming that $K$ is symmetric about the origin, integration of the numerator leads to

$$
\hat{m}_{h}(t)=\frac{\sum_{i=1}^{N} K_{h}\left(t-t_{i}\right) y_{i}}{\sum_{i=1}^{N} K_{h}\left(t-t_{i}\right)} .
$$

Intuitively, the Nadaraya-Watson estimator returns the weighted average of the observations $y_{i}$, with the weighting determined by the kernel. Note that $\hat{f}^{h, g}(t, y)$ is factored out of the estimator - the weights only depend on the values $t_{i}$.

In Figure 1 we illustrate univariate kernel regression by applying it to demonstrate the effect of aging on ventricle volume and gray matter volume in the brain. This illustration is based on data collected by Mortamet et al. [32]. Each point represents a volume measurement, relative to total brain volume, for a particular patient. These measurements were derived from 3D MR images of 50 healthy adults ranging in age from 20 to 72 using an expectation-maximization based automatic segmentation method [24]. We used kernel regression to estimate the relationship, on average, between volume and patient age (filled lines). A Nadaraya-Watson kernel estimator with a Gaussian kernel of width $\sigma=6$ years was used.

\subsection{Kernel regression on Riemannian manifolds}

In this section we consider the regression problem in the more general setting of manifold-valued observations. Let $\left\{t_{i}, p_{i}\right\}_{i=1}^{N}$ be a collection of observations where the $t_{i}$ are drawn from a univariate random variable $T$, but where $p_{i}$ are points on a Riemannian manifold $\mathcal{M}$. The classical kernel regression methods presented in Section 2.1 are not applicable in this setting because they rely on the vector space structure of the observations. In particular, the addition operator in (4) is not well defined. 
The goal is to determine the relationship, on average, between the independent variable $T$ and the distribution of the points $\left\{p_{i}\right\}$ on the manifold. This relationship can be modeled by

$$
p_{i}=\operatorname{Exp}_{m\left(t_{i}\right)}\left(\epsilon_{i}\right)
$$

where $m: \mathbb{R} \rightarrow \mathcal{M}$ defines a curve on $\mathcal{M}$. The error term $\epsilon_{i} \in T_{m\left(t_{i}\right)} \mathcal{M}$ is a tangent vector that is interpreted as the displacement along the manifold of each observation $p_{i}$ from the curve $m(t)$. The exponential mapping, Exp, returns the point on $\mathcal{M}$ at time one along the geodesic flow beginning at $m\left(t_{i}\right)$ with initial velocity $\epsilon_{i}$.

Following the univariate case, we define the regression function $m(t)$ in terms of expectation. However, in this case we generalize the idea of expectation of real random variables to manifold-valued random variables via Fréchet expectation [11,21]. Let $f(p), p \in \mathcal{M}$ be a probability density on the manifold. The Fréchet expectation is defined as

$$
\mathbb{E}_{f}[p] \equiv \underset{q \in \mathcal{M}}{\operatorname{argmin}} \int_{\mathcal{M}} d(q, p)^{2} f(p) d p
$$

where $d(q, m)$ is the metric on the manifold $\mathcal{M}$. This definition is motivated by a minimum variance characterization of the mean, where variance is defined in terms of the metric. Note that Fréchet expectation might not be unique [21]. Using the above definition, an empirical estimate of the Fréchet mean, given a collection of observations $\left\{p_{i}, i=1 \cdots N\right\}$ on a manifold $\mathcal{M}$, is defined by

$$
\mu=\underset{q \in \mathcal{M}}{\operatorname{argmin}} \frac{1}{N} \sum_{i}^{N} d\left(q, p_{i}\right)^{2} .
$$

Motivated by the definition of the Nadaraya-Watson estimator as a weighted averaging, we define a manifold kernel regression estimator using the weighted Fréchet empirical mean estimator as

$$
\hat{m}_{h}(t)=\underset{q \in \mathcal{M}}{\operatorname{argmin}}\left(\frac{\sum_{i=1}^{N} K_{h}\left(t-t_{i}\right) d\left(q, p_{i}\right)^{2}}{\sum_{i=1}^{N} K_{h}\left(t-t_{i}\right)}\right) .
$$

This estimator is illustrated in Figure 3. Notice that when the manifold under study is a Euclidean vector space, equipped with the standard Euclidean norm, the above minimization results in the Nadaraya-Watson estimator.

\subsection{Bandwidth selection}

It is well known within the kernel regression literature that kernel width plays a crucial role in determining regression results [38]. In particular, it is important to select a bandwidth that captures relevant population-wide changes without either oversmoothing and missing relevant changes or undersmoothing and biasing the results based on individual noisy data points. The 'Goldie Locks' method of tuning the bandwidth until the results are most pleasing is a common subjective method for bandwidth selection. However, non-subjective methods may be required, for example, when kernel regression is part of a larger statistical study. A number of automatic kernel bandwidth selection techniques have been proposed for this purpose $[38,16,25]$. 


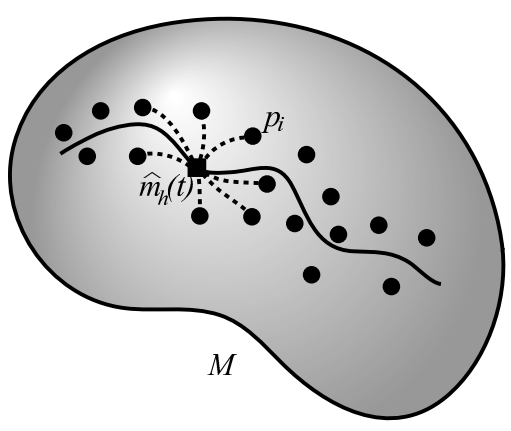

(a)

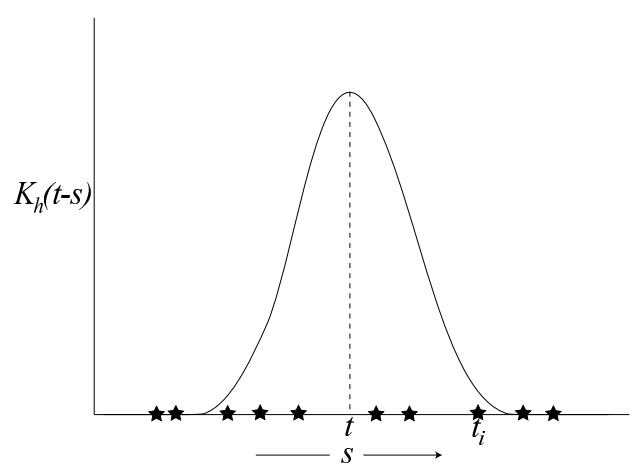

(b)

Fig. 3 Manifold kernel regression schematic. (a) For any value of the predictor variable $t$, the manifold-valued observations $p_{i}$ are summarized by the weighted Fréchet mean point $\hat{m}_{h}(t)$. (b) As in the univariate case, the weights are determined by the predictor values $t_{i}$ and the kernel $K_{h}$.

One classic method for automatic bandwidth selection is based on least squares cross-validation. This method is easily extended to the manifold regression setting in the following way. For observations $\left\{t_{i}, p_{i}\right\}_{i=1}^{N}$, with $t_{i} \in \mathbb{R}$ and $p_{i} \in \mathcal{M}$, the least squares cross-validation estimate for the optimal bandwidth $h$ is defined as

$$
\hat{h}_{\mathrm{LSCV}} \equiv \underset{h \in \mathbb{R}^{+}}{\operatorname{argmin}} \frac{1}{N} \sum_{i=1}^{N} d\left(\hat{m}_{h}^{i-}\left(t_{i}\right), p_{i}\right)^{2}
$$

where

$$
\hat{m}_{h}^{i-}(t) \equiv \underset{q \in \mathcal{M}}{\operatorname{argmin}}\left(\frac{\sum_{j=1, j \neq i}^{N} K_{h}\left(t-t_{j}\right) d\left(q, p_{j}\right)^{2}}{\sum_{j=1, j \neq i}^{N} K_{h}\left(t-t_{j}\right)}\right)
$$

is the manifold kernel regression estimator with the $i$-th observation left out.

It is important to note that equation 9 may achieve multiple local minima; this is true even in Euclidean space [14].

\subsection{Regression of rotational pose $(S O(3))$}

Before we present results of the study of brain growth, we exemplify the methodology in detail on the finite-dimensional Lie group of 3D rotations, $S O(3)$.

Following the approach in [7], we solve the weighted averaging problem in (8) by a gradient descent algorithm. The tangent space of $S O(3)$ at the identity is the Lie algebra of $3 \times 3$ skew-symmetric matrices, denoted $\mathfrak{s o}(3)$. We equip $S O(3)$ with the standard bi-invariant metric, given by the Frobenius inner product on $\mathfrak{s o}(3)$. The tangent space at an arbitrary rotation $R \in S O(3)$ is given by either left or right multiplication of $\mathfrak{s o}(3)$ by $R$.

The Lie group exponential map and its inverse, the log map, are used to compute geodesics and distances. The exponential map for a tangent vector $X \in \mathfrak{s o}(3)$ is given 
by

$$
\exp (X)= \begin{cases}I, & \theta=0, \\ I+\frac{\sin \theta}{\theta} X+\frac{1-\cos \theta}{\theta^{2}} X^{2}, & \theta \in(0, \pi),\end{cases}
$$

where $\theta=\sqrt{\frac{1}{2} \operatorname{tr}\left(X^{T} X\right)}$. A geodesic $\gamma(t)$ starting at a point $R \in S O(3)$ with initial velocity $R X$ is given by $\gamma(t)=R \exp (t X)$. The Lie group log map for a rotation matrix $R \in S O(3)$ is given by

$$
\log (R)= \begin{cases}I, & \theta=0, \\ \frac{\theta}{2 \sin \theta}\left(R-R^{T}\right), & |\theta| \in(0, \pi),\end{cases}
$$

where $\operatorname{tr}(R)=2 \cos \theta+1$. The distance between two rotations $R_{1}, R_{2} \in S O(3)$ is given by $d\left(R_{1}, R_{2}\right)=\left\|\log \left(R_{1}^{-1} R_{2}\right)\right\|$.

Now consider the weighted averaging problem with rotation data $R_{i} \in S O(3)$ and corresponding weights $w_{i}=K_{h}\left(t-t_{i}\right) / \sum_{j=1}^{N} K_{h}\left(t-t_{j}\right)$. The regression problem in (8) minimizes the weighted sum-of-squared distance function of the form $f\left(R,\left\{R_{i}, w_{i}\right\}\right)=$ $\sum_{i} w_{i} d\left(R, R_{i}\right)^{2}$. The gradient for this function at a point $R \in S O(3)$ is given by $\nabla_{R} f=$ $-\sum_{i} w_{i} R \log \left(R^{-1} R_{i}\right)$. Therefore, given the estimate $\hat{R}_{k}$ for the weighted average, the gradient descent update to solve (8) is given by $\hat{R}_{k+1}=\hat{R} \exp \left(-R^{-1} \nabla_{\hat{R}_{k}} f\right)$.

\subsection{Kernel regression for populations of brain images}

In this section we apply our shape regression methodology to study the effect of aging on brain shape from random design image data. We have observations of the form $\left\{t_{i}, I_{i}\right\}_{i=1}^{N}$ where $t_{i}$ is the age of patient $i$ and $I_{i}$ is a three-dimensional image that we identify with the anatomical configuration of patient $i$. We seek the unknown function $m$ that associates a representative anatomical configuration, and its associated image $\hat{I}$, with each age.

Let $\Omega \subset \mathbb{R}^{3}$ be the underlying coordinate system of the observed images $I_{i}$. Each image $I \in \mathcal{I}$ can be formally defined as an $L^{2}$ function from $\Omega$ to the reals. However, it is important to point out that we cannot rely on the natural $L^{2}$ structure of the images themselves for our analysis. While images can be added voxel-wise, the result is a loss of any identification with the anatomical configuration.

Instead, we represent anatomical differences in terms of transformations of the underlying image coordinates. This approach is common within the shape analysis literature $[12,29]$. Because we are interested in capturing the large, natural geometric variability evident in the brain (cf. Figure 2), we represent shape change as the action of the group of diffeomorphisms, denoted by $\mathcal{H}$. In the rest of this section, we formalize this notion and define a distance between shapes that is valid in this setting and will allow us to apply our regression methodology.

Let $\mathcal{H}$ be the group of diffeomorphisms that are isotopic to the identity. Each element $\phi: \Omega \rightarrow \Omega$ in $\mathcal{H}$ deforms an image according to the following rule

$$
I_{\phi}(x)=I\left(\phi^{-1}(x)\right) .
$$

We apply the theory of large deformation diffeomorphisms $[1,9,18,30]$ to generate deformations $\phi$ that are solutions to the Lagrangian ODEs $\frac{d}{d s} \phi_{s}(x)=v_{s}\left(\phi_{s}(x)\right)$ for a 
simulated time parameter $s \in[0,1]$. The transformations are generated by integrating the time-varying velocity fields $v_{s}$ forward in time.

We introduce a metric on $\mathcal{H}$ using a Sobelev norm via a partial differential operator $A$ applied to $v$ where $\left\|v_{s}\right\|_{V}^{2} \equiv \int_{\Omega}\left\langle A v_{s}, v_{s}\right\rangle d x$. Let $e \in \mathcal{H}$ be the identity transformation. We define the squared metric $d_{\mathcal{H}}(e, \phi)^{2}$ as

$$
d_{\mathcal{H}}(e, \phi)^{2}=\min _{v: \dot{\phi}_{s}=v_{s}\left(\phi_{s}\right)} \int_{0}^{1}\left\|v_{s}\right\|_{V}^{2} d s
$$

subject to

$$
\phi(x)=x+\int_{0}^{1} v_{s}\left(\phi_{s}(x)\right) d s \text { for all } x \in \Omega .
$$

The distance between any two diffeomorphisms is defined by

$$
d_{\mathcal{H}}\left(\phi_{1}, \phi_{2}\right)^{2}=d_{\mathcal{H}}\left(e, \phi_{1}^{-1} \circ \phi_{2}\right)^{2} .
$$

This distance satisfies all of the properties of a metric: it is non-negative, symmetric, and satisfies the triangle inequality[31].

Using this metric on $\mathcal{H}$, we can define the distance between two images as

$$
d_{\mathcal{I}}\left(I_{1}, I_{2}\right)^{2} \equiv \min _{v: \dot{\phi}_{s}=v_{s}\left(\phi_{s}\right)}\left[\int_{0}^{1}\left\|v_{s}\right\|_{V}^{2} d s+\frac{1}{\sigma^{2}}\left\|I_{1}\left(\phi^{-1}\right)-I_{2}\right\|_{L_{2}}^{2}\right]
$$

where the second term accounts for the noise model of the image [17]. While this construction is motivated by the metric on $\mathcal{H}$, it does not strictly define a Riemannian metric on the space of anatomical images (because of the second term). In the future we plan to define distance in terms of the elegant construction described in [37].

Having defined a metric on the space of images that accommodates anatomical variability, we can apply that metric to regress a representative anatomical configuration, with associated image, from our observations $\left\{t_{i}, I_{i}\right\}$

$$
\hat{I}_{h}(t)=\underset{I \in \mathcal{I}}{\operatorname{argmin}}\left(\frac{\sum_{i=1}^{N} K_{h}\left(t-t_{i}\right) d_{\mathcal{I}}\left(I, I_{i}\right)^{2}}{\sum_{i=1}^{N} K_{h}\left(t-t_{i}\right)}\right) .
$$

Equation 18 expresses the following intuitive idea: For any age $t$, the population can be represented by the anatomical configuration that is centrally located, according to $d_{\mathcal{I}}$, among the observations that occur near in time to $t$. As in the univariate case, the weights are determined by the kernel $K$.

\subsection{Diffeomorphic growth model}

Having regressed a population representative anatomical image, as a function of age, we can now study the local shape changes evident - for the population - as a function of age. We use the manifold kernel regression estimator to extend a single-subject longitudinal growth model in order to study population-average geometric change. In particular, we estimate the age-indexed diffeomorphism that quantifies the fine scale anatomical shape change of the population representative $\hat{I}$. 


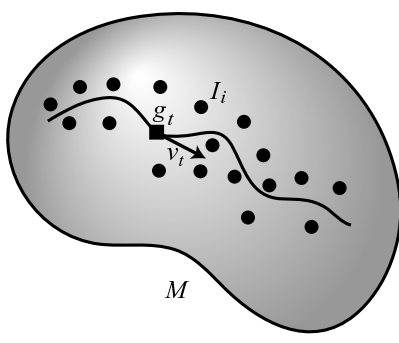

(a)

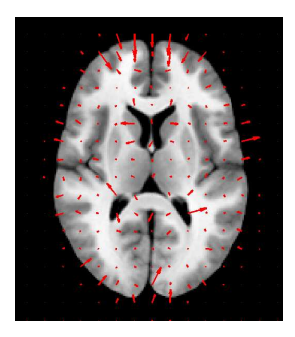

(b)

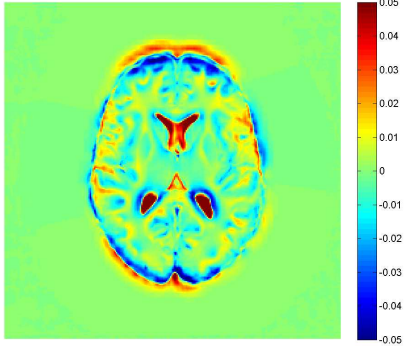

(c)

Fig. 4 Population growth model schematic. (a) The diffeomorphism $g_{t}$ quantifies the geometric change of $\hat{I}$ throughout the growth period. (b) The velocity field that is identified with the tangent vector $v_{t}=\dot{g}_{t}$ is overlaid on the underlying anatomical image $\hat{I}(t)$. The arrows indicate instantaneous shape change at age $t$. (c) This colormap identifies regions of local expansion and contraction of the underlying anatomy at time $t$. Red indicates expansion; blue indicates contraction. See text for details.

\subsubsection{Single-subject growth model}

The dynamic growth model described in [28] associates a single subject with a collection of image observations $J_{t} \in \mathcal{I}$, which are acquired over a period of time $t \in[0,1]$. The goal is to determine the diffeomorphic flow $g_{t}$ that deforms an exemplar image $J_{\alpha}$ through time in such a way that it matches these image observations. In practice $J_{0}$ is used as the exemplar image. This methodology has been applied, for example, to measure growth or atrophy of structures within the brain.

The formalization of the growth problem is similar to the definition of the image metric $d_{\mathcal{I}}$ (cf. equation 17) in that it is defined as a minimization problem that seeks to find a solution $g_{t}$ that requires the least amount of deformation according to the metric $d_{\mathcal{H}}$ on the space of diffeomorphisms:

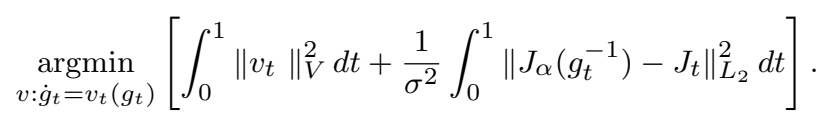

A primary difference between this equation and equation 17 is that in the case of the growth model the second term is integrated over time. This enforces the requirement that the deforming exemplar image $J_{\alpha}\left(g_{t}^{-1}\right)$ match the observed imagery $J_{t}$ throughout the growth period.

It has been shown using the calculus of variations [31] that the solution to equation 19 satisfies

$$
A v_{t}=-\frac{1}{2 \sigma^{2}} \nabla\left(J_{\alpha} \circ g_{t}^{-1}\right) \int_{t}^{1}\left(J_{u}\left(g_{u} \circ g_{t}^{-1}\right)-J_{\alpha}\left(g_{t}^{-1}\right)\right)\left|D\left(g_{u} \circ g_{t}^{-1}\right)\right| d u
$$

where $\nabla\left(J_{\alpha} \circ g_{t}^{-1}\right)$ is the gradient of the deformed exemplar image and $D\left(g_{u} \circ g_{t}^{-1}\right)$ is the Jacobian of the diffeomorphic transformation that maps the anatomical configuration at time $t$ to the configuration at time $u$. The discrete version of this equation is used to construct an iterative solution for $v_{t} . g_{t}$ is initially set to the identity map for all $t$. At each iteration $v_{t}, t \in[0,1]$ is updated according to the the observed images $J_{t}$ and the current estimate of $g_{t}, t \in[0,1]$. 


\subsubsection{Population growth model}

In order to extend this growth model to apply to a population of subjects, we replace the subject-specific collection of observed imagery $J_{t}$ with the expected observed imagery, as a function of time, for the population (cf. Figure 4). This is achieved by combining the manifold kernel regression estimator (equation 18) with the growth model (equation 19):

$$
\begin{aligned}
\underset{v: \dot{g}_{t}=v_{t}\left(g_{t}\right)}{\operatorname{argmin}} \int_{0}^{1}\left\|v_{t}\right\|_{V}^{2} d t+ & \\
& \frac{1}{\sigma^{2}} \int_{0}^{1}\left\|I_{\alpha}\left(g_{t}^{-1}\right)-\underset{I \in \mathcal{I}}{\operatorname{argmin}}\left(\frac{\sum_{i=1}^{N} K_{h}\left(t-t_{i}\right) d_{\mathcal{I}}\left(I, I_{i}\right)^{2}}{\sum_{i=1}^{N} K_{h}\left(t-t_{i}\right)}\right)\right\|_{L_{2}}^{2} d t .
\end{aligned}
$$

In this way the population representative images serve as a collection of population average time-sequence imagery.

In order to solve equation 21 we first solve the interior minimization problem for a discrete collection of time points. This is legitimate since this problem does not depend on the growth deformation $g_{t}$. Once these population representative images are computed, the time-indexed deformation $g_{t}$ is computed using the iterative method based on equation 20. We use $I_{\alpha} \equiv \hat{I}(0)$ as our population exemplar image. In order to speed convergence, we apply the growth model within a three-level multi-resolution framework where initial solutions at coarser scale levels are used to initialize the optimization procedure at finer scale levels.

Once $g_{t}$ is computed, it can be analyzed to determine local, age-indexed geometric change for the population. For example, instantaneous local growth and atrophy can be measured via the log-determinant of the Jacobian of the velocity field defined by

$$
\log \left|\begin{array}{lll}
\frac{\partial \dot{g}_{t}^{1}}{\partial x^{1}}(x) & \frac{\partial \dot{g}_{t}^{1}}{\partial x^{2}}(x) & \frac{\partial \dot{g}_{t}^{1}}{\partial x^{3}}(x) \\
\frac{\partial \dot{g}_{t}^{2}}{\partial x^{1}}(x) & \frac{\partial \dot{g}_{t}^{2}}{\partial x^{2}}(x) & \frac{\partial \dot{g}_{t}^{2}}{\partial x^{3}}(x) \\
\frac{\partial \dot{g}_{t}^{3}}{\partial x^{1}}(x) & \frac{\partial \dot{g}_{t}^{3}}{\partial x^{2}}(x) & \frac{\partial \dot{g}_{t}^{3}}{\partial x^{3}}(x)
\end{array}\right|
$$

Values of the log-Jacobian greater than zero indicate local expansion; values less than zero indicate local contraction.

\section{Results}

3.1 Synthetic data experiment

Before describing the anatomical study, we present a proof of concept experiment based on synthetic data. In this experiment, we apply our manifold regression method to a database of synthetic $2 \mathrm{D}$ images that were generated from a known, underlying geometric process. Or goal is to recover, from the imagery alone, the underlying geometric change.

The database consists of two cohorts that each contain $100256 \times 2562$ D bulls-eye images. The cohorts, $B_{1}$ and $B_{2}$, differ by the amount of random geometric variation present. Each image is associated with a particular value of the synthetic predictor variable $t \in[0,1]$; the values of $t$ for the database were drawn from a uniform random 


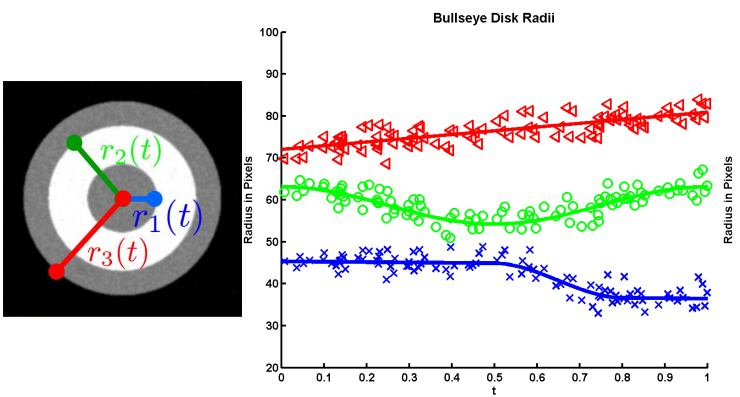

(a)

(b)

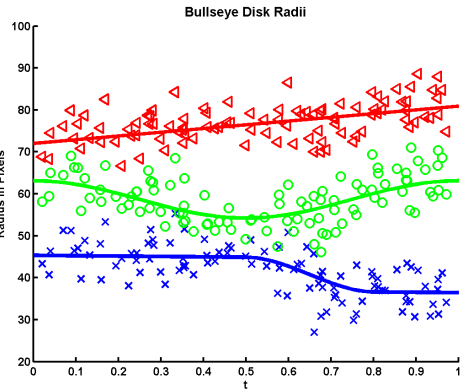

(c)

Fig. 5 Synthetic bulls-eye data set construction. The bulls-eye database contains 200 total 2D images; each images is associated with a value of $t$ drawn from a uniform distribution on $[0,1]$. (a) Images are generated from three independent, noisy radius values: $r_{1}, r_{2}$, and $r_{3}$. (b) Cohort $B_{1}$ : each observed radius value (markers) is a function of $t$ and is determined by adding random noise to the ground truth functions $f_{1}, f_{2}$, and $f_{3}$, which are depicted by the solid curves. (c) The second cohort, $B_{2}$, was generated using a higher level of random geometric variation.

distribution on $[0,1]$. For the $i$-th image there are three disks which independently change in radii according to

$$
\begin{aligned}
& r_{1}\left(t_{i}\right)=f_{1}\left(t_{i}\right)+\epsilon_{i}+\epsilon_{i, 1} \\
& r_{2}\left(t_{i}\right)=f_{2}\left(t_{i}\right)+\epsilon_{i}+\epsilon_{i, 2} \\
& r_{3}\left(t_{i}\right)=f_{3}\left(t_{i}\right)+\epsilon_{i}+\epsilon_{i, 3}
\end{aligned}
$$

subject to

$$
r_{1}(t)<r_{2}(t)<r_{3}(t) \text { for all } t \in[0,1] .
$$

The functions $f_{1}, f_{2}$, and $f_{3}$ are known; they define the noise-free, ground-truth geometric change as a function of $t$. Noise is added to these radius functions via the zero mean Gaussian random variables $\epsilon_{i}, \epsilon_{i, 1}, \epsilon_{i, 2}$ and $\epsilon_{i, 3}$. For cohort $B_{1}, \epsilon_{i} \sim$ $N\left(\mu=0, \sigma^{2}=4\right.$ pixels $)$ and $\epsilon_{i, 1}, \epsilon_{i, 2}, \epsilon_{i, 3} \sim N\left(\mu=0, \sigma^{2}=1\right.$ pixels $)$. For cohort $B_{2}$, $\epsilon_{i} \sim N\left(\mu=0, \sigma^{2}=16\right.$ pixels $)$ and $\epsilon_{i, 1}, \epsilon_{i, 2}, \epsilon_{i, 3} \sim N\left(\mu=0, \sigma^{2}=4\right.$ pixels $)$. Once the image geometries are fixed i.i.d Gaussian noise is added to the image intensities. Figure 5 contains a schematic of the image generation process. Figure 6 displays a sample of the images from this database.

For each cohort, we applied our algorithm in order to regress a population representative bulls-eye image for 8 equally spaced values of $t$. A kernel bandwidth of $\sigma=0.045$ was used. For this experiment, the solutions to equation 17 were computed using MATLAB codes based on the LDDMM algorithm described in $[2,3]$.

Figure 7 contains the results of this experiment. The regressed images are shown in the background. The ground truth radii values, $f_{1}, f_{2}$, and $f_{3}$, are depicted as colored overlays. The close agreement with the regressed images and the overlays indicates that the underlying geometric process was recovered from the image database - that is, the underlying time effect was separated from the random geometric variation. Comparing the results for the two cohorts, the regression of the geometries is rather robust to level of the geometrical noise. Only a slight degradation in accuracy of the estimate is seen with a four-fold increase in the radii noise variance. 


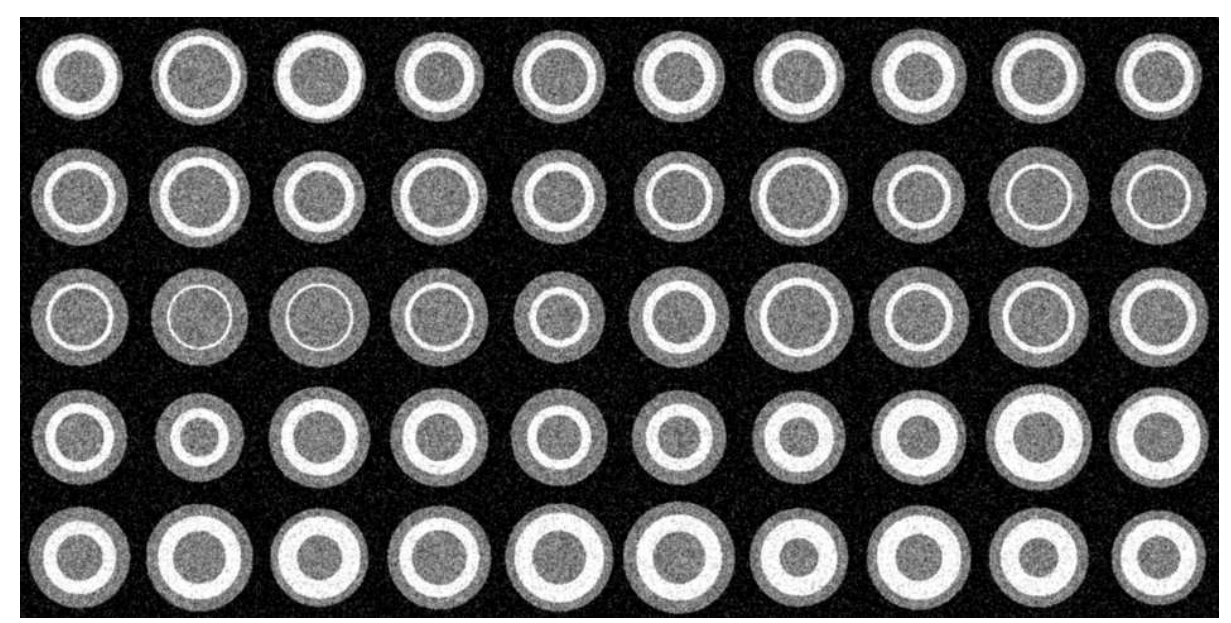

Fig. 6 Random design database of 2D bulls-eye images. These images are taken from cohort $B_{2}$. Associated time measurements increase from left to right and from top to bottom. Inner, middle, and outer disk radii are generated by adding noise to the underlying curves depicted in Figure 5 (b) and (c).

\subsection{Regressing average change of the healthy brain from 3D MR images}

To demonstrate our method for estimating cross-sectional growth, we applied the algorithm to a database of 3D MR images. The database contains MRA, T1-FLASH, T1-MPRAGE, and T2-weighted images from 97 healthy adults ranging in age from 20 to 79 [26]. For this study we only utilized the T1-FLASH images; these images were acquired at a spatial resolution of $1 \mathrm{~mm} \times 1 \mathrm{~mm} \times 1 \mathrm{~mm}$ using a 3 Tesla head-only scanner. The tissue exterior to the brain was removed using a mask generated by a brain segmentation tool described in [35]. This tool was also used for bias correction. In the final preprocessing step, all of the images were spatially aligned to an atlas using affine registration.

We applied our algorithm separately for males and females. We selected only patients for which T1-Flash data was available. The final size of the male cohort is 38 subjects ranging in age from 22 to 72 ; the final size of the female cohort is 46 subjects ranging in age from 20 to 66 . Midaxial slices for a sample of these subjects are shown in figure 2 .

We applied the manifold kernel regression estimator (18) to compute representative anatomical images for each cohort. Images were computed for ages 30 to 60 at increments of 1 year using a Gaussian kernel with $\sigma=6$ years. This bandwidth was subjectively determined. Figures 8 and 9 contain slices from these representative images.

We applied the diffeomorphic growth estimation algorithm described in Section 2.6 to determine the anatomical shape change over time for each cohort. Figure 10 illustrates the instantaneous change in the deformation at 8 different ages. More precisely, the figure shows the log-determinant of the Jacobian of the time-derivative of the deformation. In these images, red pixels indicate expansion of the underlying tissue, at the given age, while blue pixels indicate contraction. According to these determinant 

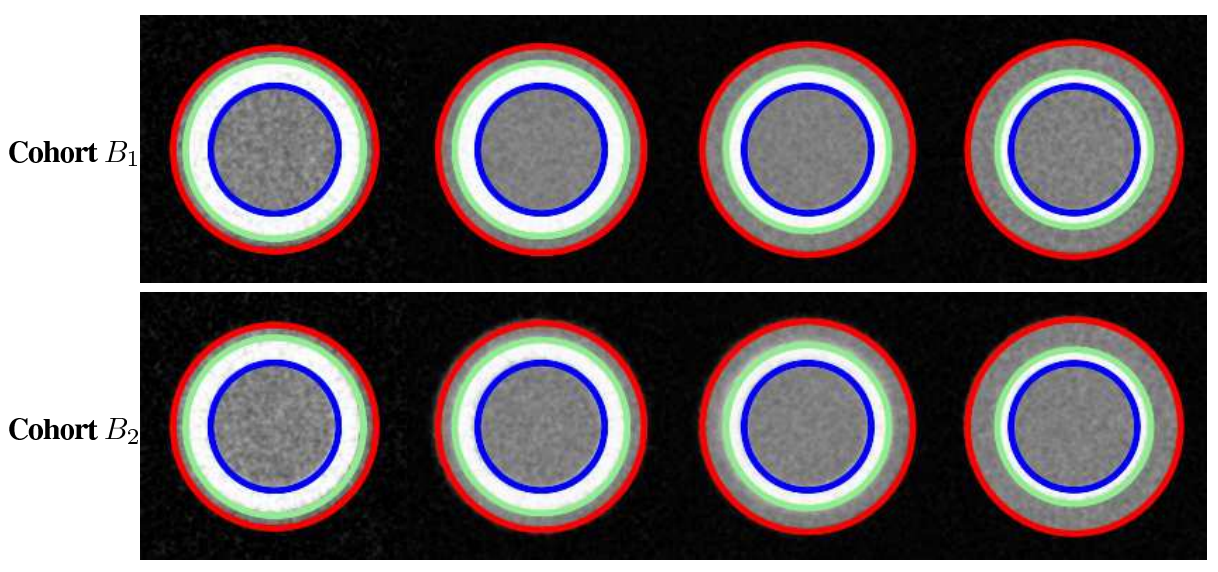

$\mathbf{t}=\mathbf{0}$

0.143

0.286

0.428
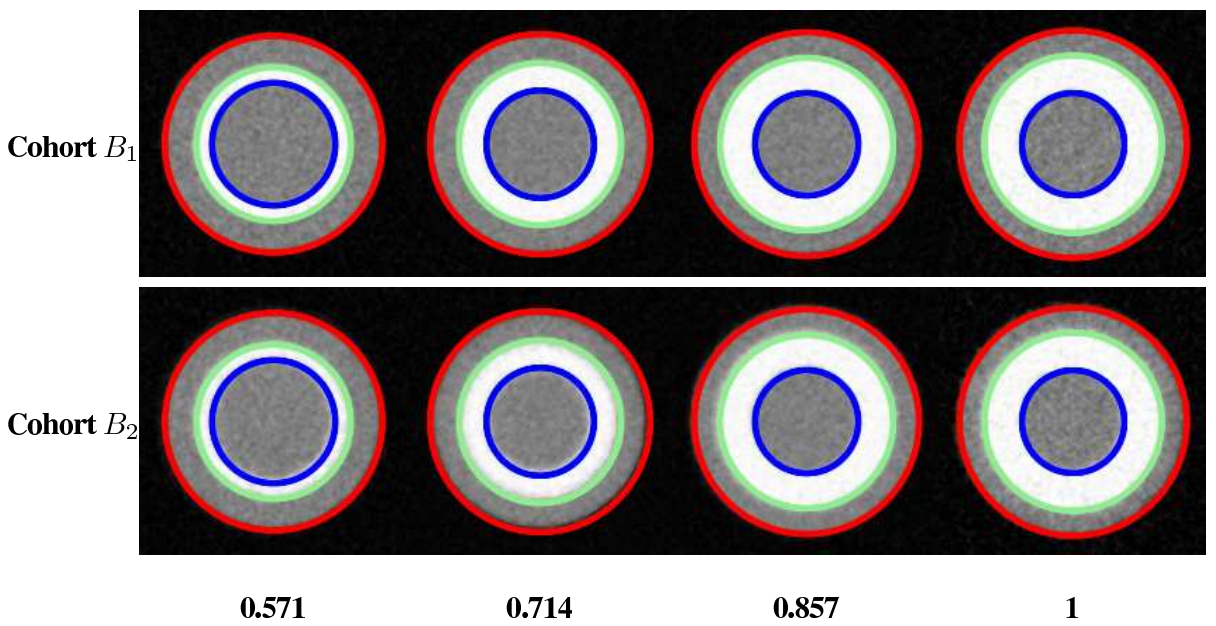

Fig. 7 Regression results for synthetic bulls-eye database. These images show the regression results for the bulls-eye database at 8 equally-spaced time points for cohorts $B_{1}$ and $B_{2}$. Colored overlays denote the ground truth radii as determined by the underlying curves in Figure $6(\mathrm{~b})$ and (c).

maps, expansion of the ventricles is evident for each age group. However, the expansion is accelerated for ages 50 to 60 . Note that this finding agrees well with volume-based regression analysis from Figure 1.

\subsubsection{Computational Strategy}

For this study we approximate solutions to (18) using an iterative greedy algorithm that is similar to the method described in [17]. Results were computed using a multithreaded $\mathrm{C}++$ implementation on an 8 processor (16 core) $3 \mathrm{GHz}$ system with approximately 64 gigabytes of RAM. Processing time averaged 116 minutes per $256 \times 256 \times 256$ regressed image volume. 

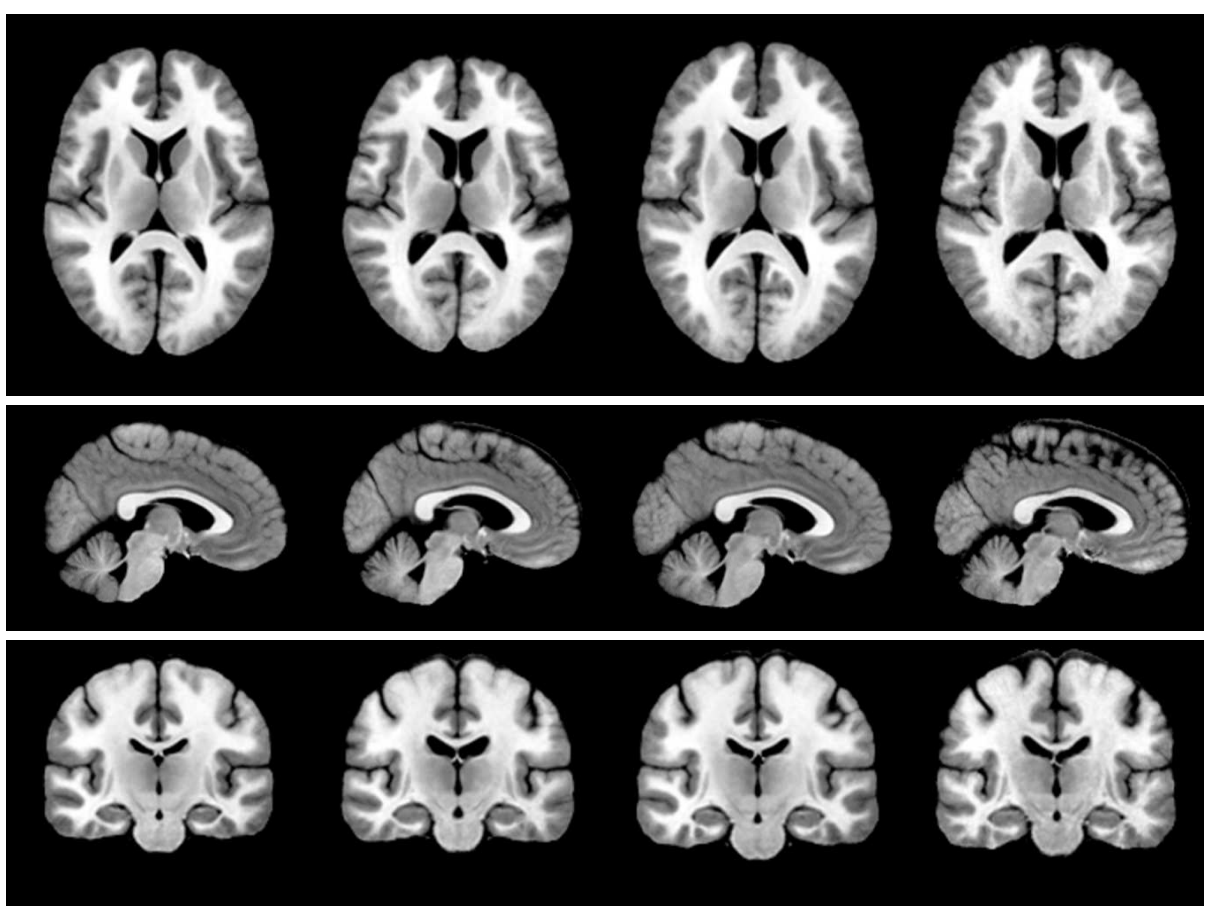

Female age $=30$

Female age $=60$

Male age $=\mathbf{3 0}$

Male age $=60$

Fig. 8 Regressed brain images. Representative anatomical images for each cohort at ages 30 (left) and 60 (right). These images were generated from the random design 3D MR database using the shape regression method described in Section 2.

When computing each representative image $\hat{I}(x)$, we use a multi-resolution approach that generates images at progressively higher resolutions, where each level is initialized by the results at the next coarsest scale. This strategy has the dual benefits of (a) addressing the large scale shape changes first and (b) speeding algorithm convergence.

The dominating computation at each iteration is a Fast Fourier Transform. The order of the algorithm is $M N n \log n$ where $M$ is the number of iterations, $N$ is the number of images, and $n$ is the number of voxels along the largest dimension of the images. Therefore, the complexity grows linearly with the number of observations, making this algorithm suitable for application to large data sets.

\section{Conclusion}

We have proposed a method for population shape regression that enables novel analysis of population shape and growth from random design data when the underlying shape model is non-Euclidean. While the method is quite general, in this paper we apply this method to study the effect of aging on the brain. We regress a population representative shape, indexed by age, from a database of MR brain images. Finally, we apply a 


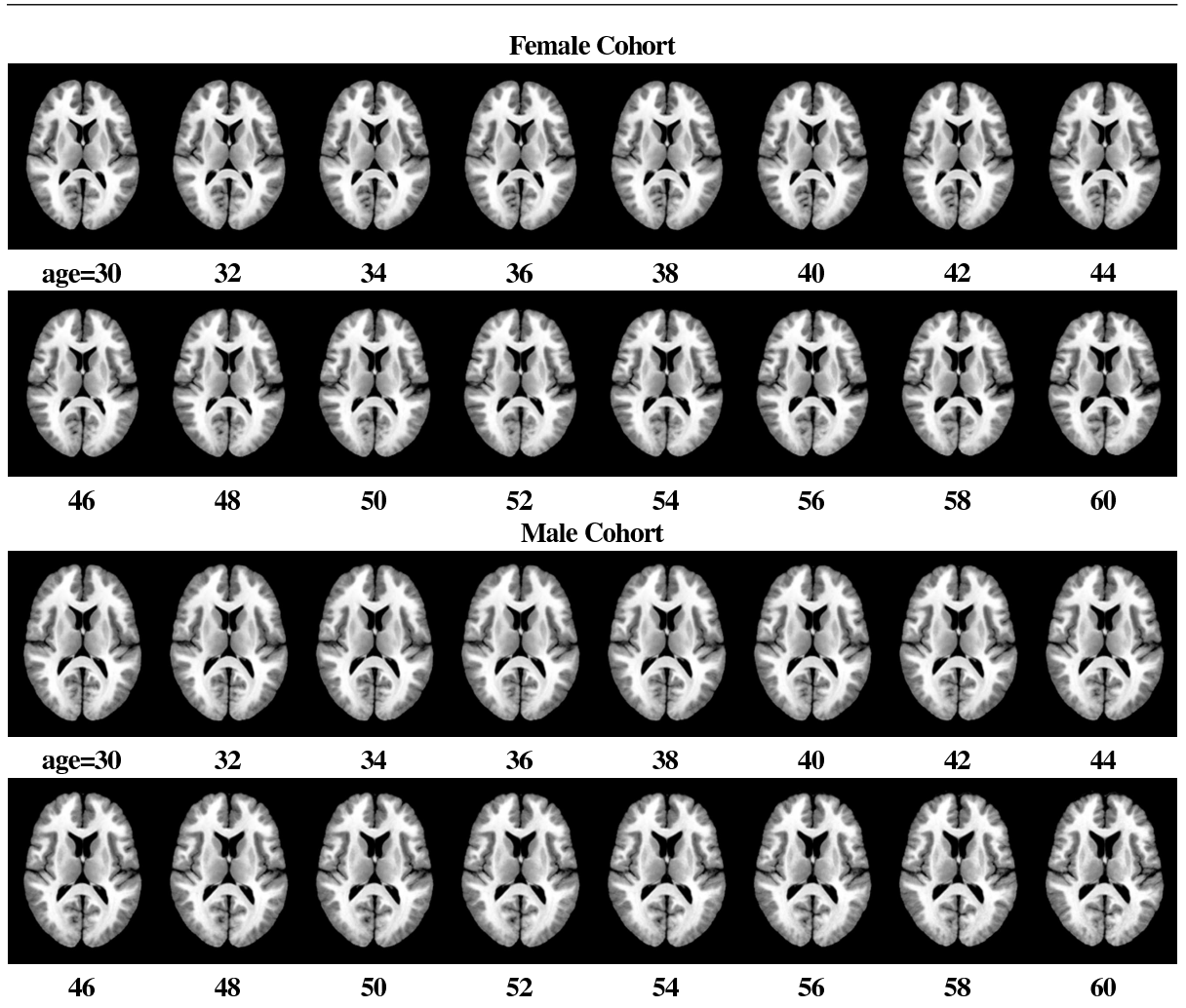

Fig. 9 The average, aging brain. These images show the average brain shape as a function of age for the female (top) and male (bottom) cohorts. These are not images from any particular patient - they are computed using the regression method proposed in this paper (18). Noticeable expansion of the lateral ventricles is clearly captured in both the image data and the determinant maps (Figure 10). All 2D slices are extracted from the $3 \mathrm{D}$ volumes that were used for computation.

longitudinal growth model to these representative images to study the detailed local shape change that occurs, on average, as a function of age.

Acknowledgements We thank Bénédicte Mortamet for providing tissue volume data and Peter Lorenzen for image preprocessing. We gratefully acknowledge our funding sources including NIH grants R01 EB000219-NIH-NIBIB, and R01 CA124608-NIH-NCI.

\section{References}

1. Beg, M., Miller, M., Trouvé, A., Younes, L.: Computing large deformation mettric mappings via geodesic flows of diffeomorphisms. International Journal of Computer Vision 61(2) (2005)

2. Beg, M.F.: Variational and computational methods for flows of diffeomorphisms in image matching and growth in computational anatomy. PhD thesis, The Johns Hopkins University (2003)

3. Beg, M.F., Miller, M.I., Trouvé, A., Younes, L.: Computing large deformation metric mappings via geodesic flows of diffeomorphisms. International Journal of Computer Vision 61(2), 139-157 (2005) 


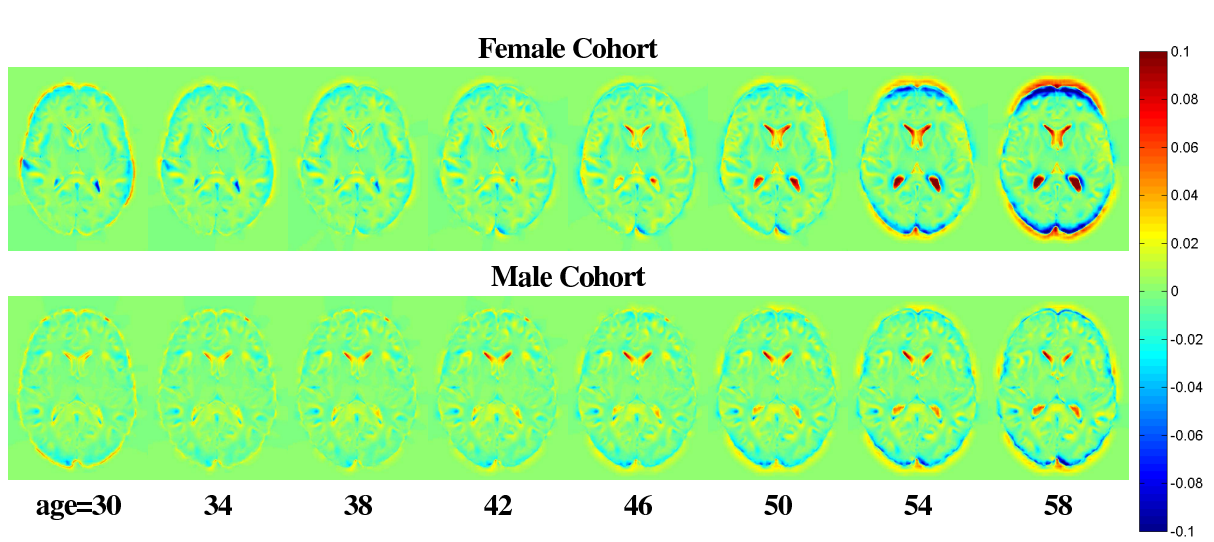

Fig. 10 Age-indexed local expansion and contraction. Illustration of the local brain shape change as a function of age for the female (top) and male (bottom) cohorts. These data were generated by applying a diffeomorphic growth 21 model to the representative images that were computed using manifold regression. Red voxels indicate local expansion; blue voxels indicate local contraction.

4. Bhattacharya, R., Patrangenaru, V.: Nonparametric estimation of location and dispersion on riemannian manifolds. Journal of Statistical Planning and Inference 108, 23-36 (2002)

5. Bhattacharya, R., Patrangenaru, V.: Large sample theory of intrinsic and extrinsic sample means on manifolds i. Annals of Statistics 31(1), 1-29 (2003)

6. Bingham, C.: An antipodally symmetric distribution on the sphere. The Annals of Statistics 2(6), 1201-1225 (1974)

7. Buss, S.R., Fillmore, J.P.: Spherical averages and applications to spherical splines and interpolation. ACM Transactions on Graphics 20(2), 95-126 (2001)

8. Clatz, O., Sermesant, M., Bondiau, P.Y., Delingette, H., Warfield, S.K., Malandain, G., Ayache, N.: Realistic simulation of the 3D growth of brain tumors in MR images coupling diffusion with mass effect. IEEE Transactions on Medical Imaging 24(10), 1334-1346 (2005)

9. Dupuis, P., Grenander, U.: Variational problems on flows of diffeomorphisms for image matching. Quarterly of Applied Mathematics LVI(3), 587-600 (1998)

10. Fletcher, P.T., Joshi, S., Ju, C., Pizer, S.M.: Principal geodesic analysis for the study of nonliner statistics of shape. IEEE Transactions on Medical Imaging 23(8), 995-1005 (2004)

11. Fréchet, M.: Les elements aleatoires de nature quelconque dans un espace distancie. Annales De L'Institut Henri Poincare 10, 215-310 (1948)

12. Grenander, U., Miller, M.I.: Computational anatomy: An emerging discipline. Quarterly of Applied Mathematics 56(4), 617-694 (1998)

13. Guttmann, C., Jolesz, F., Kikinis, R., Killiany, R., Moss, M., Sandor, T., Albert, M.: White matter changes with normal aging. Neurology 50(4), 972-978 (1998)

14. Hall, P., Marron, J.S.: Local minima in cross-validation functions. Journal of the Royal Statistical Society (Series B) 53(1), 245-252 (1991)

15. Hardle, W.: Applied Nonparametric Regression. Cambridge University Press (1990)

16. Jones, M.C., Marron, J.S., Sheather, S.J.: A brief survey of bandwidth selection for density estimation. Journal of the American Statistical Association 91(433), 401-407 (1996)

17. Joshi, S., Davis, B., Jomier, M., Gerig, G.: Unbiased diffeomorphic atlas construction for computational anatomy. NeuroImage (Supplemental issue on Mathematics in Brain Imaging) 23, S151-S160 (2004)

18. Joshi, S.C., Miller, M.I.: Landmark matching via large deformation diffeomorphisms. IEEE Transactions On Image Processing 9(8), 1357-1370 (2000)

19. Jupp, P., Mardia, K.: A unified view of the theory of directional statistics, 1975-1988. International Statistical Review 57(3), 261-294 (1989)

20. Jupp, P.E., Kent, J.T.: Fitting smooth paths to spherical data. Applied Statistics 36(1), 34-46 (1987) 
21. Karcher, H.: Riemannian center of mass and mollifier smoothing. Communications on Pure and Applied Mathematics 30, 509-541 (1977)

22. Kendall, D.G.: Shape manifolds, Procrustean metrics, and complex projective spaces. Bulletin of the London Mathematical Society 16, 18-121 (1984)

23. Le, H., Kendall, D.: The riemannian structure of euclidean shape spaces: A novel environment for statistics. The Annals of Statistics 21(3), 1225-1271 (1993)

24. Leemput, K.V., Maes, F., Vandermeulen, D., Suetens, P.: Automated model-based tissue classification of $\mathrm{mr}$ images of the brain. IEEE Transactions on Medical Imaging 18(10), 897-908 (1999)

25. Loader, C.R.: Bandwidth selection: Classical or plug-in? The Annals of Statistics 27(2), 415-438 (1999)

26. Lorenzen, P., Prastawa, M., Davis, B., Gerig, G., Bullitt, E., Joshi, S.: Multi-modal image set registration and atlas formation. Medical Image Analysis 10(3), 440-451 (2006)

27. Matsumae, M., Kikinis, R., Mórocz, I., Lorenzo, A., Sándor, T., Sándor, T., Albert, M., Black, P., Jolesz, F.: Age-related changes in intracranial compartment volumes in normal adults assessed by magnetic resonance imaging. J Neurosurg 84, 982-991 (1996)

28. Miller, M.: Computational anatomy: shape, growth, and atrophy comparison via diffeomorphisms. NeuroImage 23, S19-S33 (2004)

29. Miller, M., Banerjee, A., Christensen, G., Joshi, S., Khaneja, N., Grenander, U., Matejic, L.: Statistical methods in computational anatomy. Statistical Methods in Medical Research 6, 267-299 (1997)

30. Miller, M., Younes, L.: Group actions, homeomorphisms, and matching: A general framework. International Journal of Computer Vision 41, 61-84 (2001)

31. Miller, M.I., Trouve, A., Younes, L.: On the metrics and euler-lagrange equations of computational anatomy. Annual Review of Biomedical Engineering 4, 375-405 (2002)

32. Mortamet, B., Zeng, D., Gerig, G., Prastawa, M., Bullitt, E.: Effects of healthy aging measured by intracranial compartment volumes using a designed mr brain database. In: Lecture Notes in Computer Science (LNCS), vol. 3749, pp. 383-391. Medical Image Computing and Computer Assisted Intervention (MICCAI) (2005)

33. Nadaraya, E.A.: On estimating regression. Theory of Probability and its Applications 10, 186-190 (1964)

34. Pennec, X.: Intrinsic statistics on riemannian manifolds: Basic tools for geometric measurements. Journal of Mathematical Imaging and Vision 25, 127-154 (2006)

35. Prastawa, M., Bullitt, E., Ho, S., Gerig, G.: A brain tumor segmentation framework based on outlier detection. Medical Image Analysis 8(3), 275-283 (2004)

36. Thompson, P.M., Giedd, J.N., Woods, R.P., MacDonald, D., Evans, A.C., Toga, A.W.: Growth patterns in the developing brain detected by using continuum mechanical tensor maps. Nature 404(6774), 190-193 (2000). DOI 10.1038/35004593. URL http://dx.doi.org/10.1038/35004593

37. Trouvé, A., Younes, L.: Metamorphoses through lie group action. Foundations of Computational Mathematics 5(2), 173-198 (2005)

38. Wand, M.P., Jones, M.C.: Kernel Smoothing. No. 60 in Monographs on Statistics and Applied Probabilitiy. Chapman \& Hall/CRC (1995)

39. Watson, G.S.: Smooth regression analysis. Sankhya 26, 101-116 (1964) 